\title{
Padé approximant related to the Wallis formula
}

\author{
Long Lin', Wen-Cheng $\mathrm{Ma}^{2}$ and Chao-Ping Chen ${ }^{\text {* }}$
}

"Correspondence:
chenchaoping@sohu.com
1School of Mathematics and
Informatics, Henan Polytechnic
University, Jiaozuo City, Henan
Province 454000, China
Full list of author information is
available at the end of the article

\section{Abstract}

Based on the Pade approximation method, in this paper we determine the coefficients $a_{j}$ and $b_{j}$ such that

$$
\pi=\left(\frac{(2 n) ! !}{(2 n-1) ! !}\right)^{2}\left\{\frac{n^{k}+a_{1} n^{k-1}+\cdots+a_{k}}{n^{k+1}+b_{1} n^{k}+\cdots+b_{k+1}}+O\left(\frac{1}{n^{2 k+3}}\right)\right\}, \quad n \rightarrow \infty,
$$

where $k \geq 0$ is any given integer. Based on the obtained result, we establish a more accurate formula for approximating $\pi$, which refines some known results.

MSC: Primary 33B15; secondary 26D07; 41A60

Keywords: gamma function; psi function; Wallis ratio; inequality; approximation

\section{Introduction}

It is well known that the number $\pi$ satisfies the following inequalities:

$$
\frac{2}{2 n+1}\left(\frac{(2 n) ! !}{(2 n-1) ! !}\right)^{2}<\pi<\frac{1}{n}\left(\frac{(2 n) ! !}{(2 n-1) ! !}\right)^{2}, \quad n \in \mathbb{N}:=\{1,2,3, \ldots\},
$$

where

$$
(2 n) ! !=2 \cdot 4 \cdot 6 \cdots(2 n)=2^{n} n !, \quad(2 n-1) ! !=1 \cdot 3 \cdot 5 \cdots(2 n-1) .
$$

This result is due to Wallis (see [1]).

Based on a basic theorem in mathematical statistics concerning unbiased estimators with minimum variance, Gurland [1] yielded a closer approximation to $\pi$ than that afforded by (1.1), namely,

$$
\frac{4 n+3}{(2 n+1)^{2}}\left(\frac{(2 n) ! !}{(2 n-1) ! !}\right)^{2}<\pi<\frac{4}{4 n+1}\left(\frac{(2 n) ! !}{(2 n-1) ! !}\right)^{2}, \quad n \in \mathbb{N} .
$$

By using (1.2), Brutman [2] and Falaleev [3] established estimates of the Landau constants.

\section{Springer}

(c) The Author(s) 2017. This article is distributed under the terms of the Creative Commons Attribution 4.0 International License (http://creativecommons.org/licenses/by/4.0/), which permits unrestricted use, distribution, and reproduction in any medium, provided you give appropriate credit to the original author(s) and the source, provide a link to the Creative Commons license, and indicate if changes were made. 
Mortici [4], Theorem 2, improved Gurland's result (1.2) and obtained the following double inequality:

$$
\begin{aligned}
& \left(\frac{n+\frac{1}{4}}{n^{2}+\frac{1}{2} n+\frac{3}{32}}+\frac{9}{2,048 n^{5}}-\frac{45}{8,192 n^{6}}\right)\left(\frac{(2 n) ! !}{(2 n-1) ! !}\right)^{2} \\
& \quad<\pi<\left(\frac{n+\frac{1}{4}}{n^{2}+\frac{1}{2} n+\frac{3}{32}}+\frac{9}{2,048 n^{5}}\right)\left(\frac{(2 n) ! !}{(2 n-1) ! !}\right)^{2}, \quad n \in \mathbb{N} .
\end{aligned}
$$

We see from (1.3) that

$$
\pi=\left(\frac{(2 n) ! !}{(2 n-1) ! !}\right)^{2}\left\{\frac{n+\frac{1}{4}}{n^{2}+\frac{1}{2} n+\frac{3}{32}}+O\left(\frac{1}{n^{5}}\right)\right\}, \quad n \rightarrow \infty .
$$

Based on the Padé approximation method, in this paper we develop the approximation formula (1.4) to produce a general result. More precisely, we determine the coefficients $a_{j}$ and $b_{j}$ such that

$$
\pi=\left(\frac{(2 n) ! !}{(2 n-1) ! !}\right)^{2}\left\{\frac{n^{k}+a_{1} n^{k-1}+\cdots+a_{k}}{n^{k+1}+b_{1} n^{k}+\cdots+b_{k+1}}+O\left(\frac{1}{n^{2 k+3}}\right)\right\}, \quad n \rightarrow \infty,
$$

where $k \geq 0$ is any given integer. Based on the obtained result, we establish a more accurate formula for approximating $\pi$, which refines some known results.

The numerical values given in this paper have been calculated via the computer program MAPLE 13.

\section{Lemmas}

Euler's gamma function $\Gamma(x)$ is one of the most important functions in mathematical analysis and has applications in diverse areas. The logarithmic derivative of $\Gamma(x)$, denoted by $\psi(x)=\Gamma^{\prime}(x) / \Gamma(x)$, is called the psi (or digamma) function.

The following lemmas are required in the sequel.

Lemma 2.1 ([5]) Let $r \neq 0$ be a given real number and $\ell \geq 0$ be a given integer. The following asymptotic expansion holds:

$$
\frac{\Gamma(x+1)}{\Gamma\left(x+\frac{1}{2}\right)} \sim \sqrt{x}\left(1+\sum_{j=1}^{\infty} \frac{p_{j}}{x^{j}}\right)^{x^{\ell} / r}, \quad x \rightarrow \infty,
$$

with the coefficients $p_{j} \equiv p_{j}(\ell, r)(j \in \mathbb{N})$ given by

$$
p_{j}=\sum \frac{r^{k_{1}+k_{2}+\cdots+k_{j}}}{k_{1} ! k_{2} ! \cdots k_{j} !}\left(\frac{\left(2^{2}-1\right) B_{2}}{1 \cdot 1 \cdot 2^{2}}\right)^{k_{1}}\left(\frac{\left(2^{4}-1\right) B_{4}}{2 \cdot 3 \cdot 2^{4}}\right)^{k_{2}} \cdots\left(\frac{\left(2^{2 j}-1\right) B_{2 j}}{j(2 j-1) 2^{2 j}}\right)^{k_{j}} \text {, }
$$

where $B_{j}$ are the Bernoulli numbers summed over all nonnegative integers $k_{j}$ satisfying the equation

$$
(1+\ell) k_{1}+(3+\ell) k_{2}+\cdots+(2 j+\ell-1) k_{j}=j .
$$


In particular, setting $(\ell, r)=(0,-2)$ in $(2.1)$ yields

$$
x\left(\frac{\Gamma\left(x+\frac{1}{2}\right)}{\Gamma(x+1)}\right)^{2} \sim 1+\sum_{j=1}^{\infty} \frac{c_{j}}{x^{j}}, \quad x \rightarrow \infty
$$

where the coefficients $c_{j} \equiv p_{j}(0,-2)(j \in \mathbb{N})$ are given by

$$
c_{j}=\sum \frac{(-2)^{k_{1}+k_{2}+\cdots+k_{j}}}{k_{1} ! k_{2} ! \cdots k_{j} !}\left(\frac{\left(2^{2}-1\right) B_{2}}{1 \cdot 1 \cdot 2^{2}}\right)^{k_{1}}\left(\frac{\left(2^{4}-1\right) B_{4}}{2 \cdot 3 \cdot 2^{4}}\right)^{k_{2}} \cdots\left(\frac{\left(2^{2 j}-1\right) B_{2 j}}{j(2 j-1) 2^{2 j}}\right)^{k_{j}}
$$

summed over all nonnegative integers $k_{j}$ satisfying the equation

$$
k_{1}+3 k_{2}+\cdots+(2 j-1) k_{j}=j .
$$

Lemma $2.2([5])$ Let $m, n \in \mathbb{N}$. Then, for $x>0$,

$$
\begin{aligned}
\sum_{j=1}^{2 m}\left(1-\frac{1}{2^{2 j}}\right) \frac{2 B_{2 j}}{(2 j) !} \frac{(2 j+n-2) !}{x^{2 j+n-1}} & <(-1)^{n}\left(\psi^{(n-1)}(x+1)-\psi^{(n-1)}\left(x+\frac{1}{2}\right)\right)+\frac{(n-1) !}{2 x^{n}} \\
& <\sum_{j=1}^{2 m-1}\left(1-\frac{1}{2^{2 j}}\right) \frac{2 B_{2 j}}{(2 j) !} \frac{(2 j+n-2) !}{x^{2 j+n-1}} .
\end{aligned}
$$

In particular, we have

$$
U(x)<\psi(x+1)-\psi\left(x+\frac{1}{2}\right)<V(x)
$$

where

$$
\begin{aligned}
V(x)= & \frac{1}{2 x}-\frac{1}{8 x^{2}}+\frac{1}{64 x^{4}}-\frac{1}{128 x^{6}}+\frac{17}{2,048 x^{8}}-\frac{31}{2,048 x^{10}}+\frac{691}{16,384 x^{12}} \\
& -\frac{5,461}{32,768 x^{14}}+\frac{929,569}{1,048,576 x^{16}}
\end{aligned}
$$

and

$$
U(x)=V(x)-\frac{3,202,291}{524,288 x^{18}}
$$

For our later use, we introduce Padé approximant (see [6-11]). Let $f$ be a formal power series

$$
f(t)=c_{0}+c_{1} t+c_{2} t^{2}+\cdots
$$

The Padé approximation of order $(p, q)$ of the function $f$ is the rational function, denoted by

$$
[p / q]_{f}(t)=\frac{\sum_{j=0}^{p} a_{j} t^{j}}{1+\sum_{j=1}^{q} b_{j} t^{j}}
$$


where $p \geq 0$ and $q \geq 1$ are two given integers, the coefficients $a_{j}$ and $b_{j}$ are given by (see $[6-8,10,11])$

$$
\left\{\begin{array}{l}
a_{0}=c_{0}, \\
a_{1}=c_{0} b_{1}+c_{1}, \\
a_{2}=c_{0} b_{2}+c_{1} b_{1}+c_{2}, \\
\vdots \\
a_{p}=c_{0} b_{p}+\cdots+c_{p-1} b_{1}+c_{p}, \\
0=c_{p+1}+c_{p} b_{1}+\cdots+c_{p-q+1} b_{q}, \\
\vdots \\
0=c_{p+q}+c_{p+q-1} b_{1}+\cdots+c_{p} b_{q},
\end{array}\right.
$$

and the following holds:

$$
[p / q]_{f}(t)-f(t)=O\left(t^{p+q+1}\right)
$$

Thus, the first $p+q+1$ coefficients of the series expansion of $[p / q]_{f}$ are identical to those of $f$. Moreover, we have (see [9])

$$
[p / q]_{f}(t)=\frac{\left|\begin{array}{cccc}
t^{q} f_{p-q}(t) & t^{q-1} f_{p-q+1}(t) & \cdots & f_{p}(t) \\
c_{p-q+1} & c_{p-q+2} & \cdots & c_{p+1} \\
\vdots & \vdots & \ddots & \vdots \\
c_{p} & c_{p+1} & \cdots & c_{p+q}
\end{array}\right|}{\left|\begin{array}{cccc}
t^{q} & t^{q-1} & \cdots & 1 \\
c_{p-q+1} & c_{p-q+2} & \cdots & c_{p+1} \\
\vdots & \vdots & \ddots & \vdots \\
c_{p} & c_{p+1} & \cdots & c_{p+q}
\end{array}\right|}
$$

with $f_{n}(x)=c_{0}+c_{1} x+\cdots+c_{n} x^{n}$, the $n$th partial sum of the series $f$ in (2.7).

\section{Main results}

Let

$$
f(x)=x\left(\frac{\Gamma\left(x+\frac{1}{2}\right)}{\Gamma(x+1)}\right)^{2}
$$

It follows from (2.3) that, as $x \rightarrow \infty$,

$$
\begin{aligned}
f(x) \sim \sum_{j=0}^{\infty} \frac{c_{j}}{x^{j}}= & 1-\frac{1}{4 x}+\frac{1}{32 x^{2}}+\frac{1}{128 x^{3}}-\frac{5}{2,048 x^{4}}-\frac{23}{8,192 x^{5}}+\frac{53}{65,536 x^{6}} \\
& +\frac{593}{262,144 x^{7}}-\cdots,
\end{aligned}
$$

with the coefficients $c_{j}$ given by (2.4). In what follows, the function $f$ is given in (3.1). 
Based on the Padé approximation method, we now give a derivation of formula (1.4). To this end, we consider

$$
[1 / 2]_{f}(x)=\frac{\sum_{j=0}^{1} a_{j} x^{-j}}{1+\sum_{j=1}^{2} b_{j} x^{-j}} .
$$

Noting that

$$
c_{0}=1, \quad c_{1}=-\frac{1}{4}, \quad c_{2}=\frac{1}{32}, \quad c_{3}=\frac{1}{128}
$$

holds, we have, by (2.9),

$$
\left\{\begin{array}{l}
a_{0}=1 \\
a_{1}=b_{1}-\frac{1}{4}, \\
0=\frac{1}{32}-\frac{1}{4} b_{1}+b_{2}, \\
0=\frac{1}{128}+\frac{1}{32} b_{1}-\frac{1}{4} b_{2},
\end{array}\right.
$$

that is,

$$
a_{0}=1, \quad a_{1}=\frac{1}{4}, \quad b_{1}=\frac{1}{2}, \quad b_{2}=\frac{3}{32} .
$$

We thus obtain that

$$
[1 / 2]_{f}(x)=\frac{1+\frac{1}{4 x}}{1+\frac{1}{2 x}+\frac{3}{32 x^{2}}},
$$

and we have, by (2.10),

$$
x\left(\frac{\Gamma\left(x+\frac{1}{2}\right)}{\Gamma(x+1)}\right)^{2}-\frac{1+\frac{1}{4 x}}{1+\frac{1}{2 x}+\frac{3}{32 x^{2}}}=O\left(\frac{1}{x^{4}}\right), \quad x \rightarrow \infty .
$$

Noting that

$$
\frac{\Gamma\left(n+\frac{1}{2}\right)}{\Gamma(n+1)}=\sqrt{\pi} \cdot \frac{(2 n-1) ! !}{(2 n) ! !}, \quad n \in \mathbb{N} \text { (the Wallis ratio) }
$$

holds, replacing $x$ by $n$ in (3.4) yields (1.4).

From the Padé approximation method introduced in Section 2 and the asymptotic expansion (3.2), we obtain a general result given by Theorem 3.1. As a consequence, we obtain (1.5).

Theorem 3.1 The Pade approximation of order $(p, q)$ of the asymptotic formula of the function $f(x)=x\left(\frac{\Gamma\left(x+\frac{1}{2}\right)}{\Gamma(x+1)}\right)^{2}$ (at the point $\left.x=\infty\right)$ is the following rational function:

$$
[p / q]_{f}(x)=\frac{1+\sum_{j=1}^{p} a_{j} x^{-j}}{1+\sum_{j=1}^{q} b_{j} x^{-j}}=x\left(\frac{x^{p}+a_{1} x^{p-1}+\cdots+a_{p}}{x^{q}+b_{1} x^{q-1}+\cdots+b_{q}}\right)
$$


where $p \geq 0$ and $q \geq 1$ are two given integers and $q=p+1$ (an empty sum is understood to be zero), the coefficients $a_{j}$ and $b_{j}$ are given by

$$
\left\{\begin{array}{l}
a_{1}=b_{1}+c_{1}, \\
a_{2}=b_{2}+c_{1} b_{1}+c_{2}, \\
\vdots \\
a_{p}=b_{p}+\cdots+c_{p-1} b_{1}+c_{p}, \\
0=c_{p+1}+c_{p} b_{1}+\cdots+c_{p-q+1} b_{q}, \\
\vdots \\
0=c_{p+q}+c_{p+q-1} b_{1}+\cdots+c_{p} b_{q},
\end{array}\right.
$$

and $c_{j}$ is given in (2.4), and the following holds:

$$
f(x)-[p / q]_{f}(x)=O\left(\frac{1}{x^{p+q+1}}\right), \quad x \rightarrow \infty .
$$

Moreover, we have

$$
[p / q]_{f}(x)=\frac{\left|\begin{array}{ccccc}
\frac{1}{x^{q}} f_{p-q}(x) & \frac{1}{x^{q-1}} f_{p-q+1}(t) & \cdots & f_{p}(t) \\
c_{p-q+1} & c_{p-q+2} & \cdots & c_{p+1} \\
\vdots & \vdots & \ddots & \vdots \\
c_{p} & c_{p+1} & \cdots & c_{p+q}
\end{array}\right|}{\left|\begin{array}{cccc}
\frac{1}{x^{q}} & \frac{1}{x^{q-1}} & \cdots & 1 \\
c_{p-q+1} & c_{p-q+2} & \cdots & c_{p+1} \\
\vdots & \vdots & \ddots & \vdots \\
c_{p} & c_{p+1} & \cdots & c_{p+q}
\end{array}\right|}
$$

with $f_{n}(x)=\sum_{j=0}^{n} \frac{c_{j}}{x^{j}}$, the $n$th partial sum of the asymptotic series (3.2).

Remark 3.1 Using (3.9), we can also derive (3.3). Indeed, we have

$$
[1 / 2]_{f}(x)=\frac{\left|\begin{array}{ccc}
\frac{1}{x^{2}} f_{-1}(x) & \frac{1}{x} f_{0}(x) & f_{1}(x) \\
c_{0} & c_{1} & c_{2} \\
c_{1} & c_{2} & c_{3}
\end{array}\right|}{\left|\begin{array}{llll}
\frac{1}{x^{2}} & \frac{1}{x} & 1 \\
c_{0} & c_{1} & c_{2} \\
c_{1} & c_{2} & c_{3}
\end{array}\right|}=\frac{\left|\begin{array}{ccc}
0 & \frac{1}{x} & 1-\frac{1}{4 x} \\
1 & -\frac{1}{4} & \frac{1}{32} \\
-\frac{1}{4} & \frac{1}{32} & \frac{1}{128}
\end{array}\right|}{\left|\begin{array}{ccc}
\frac{1}{x^{2}} & \frac{1}{x} & 1 \\
1 & -\frac{1}{4} & \frac{1}{32} \\
-\frac{1}{4} & \frac{1}{32} & \frac{1}{128}
\end{array}\right|}=\frac{1+\frac{1}{4 x}}{1+\frac{1}{2 x}+\frac{3}{32 x^{2}}} .
$$

Replacing $x$ by $n$ in (3.8) applying (3.5), we obtain the following corollary.

Corollary 3.1 As $n \rightarrow \infty$,

$$
\pi=\left(\frac{(2 n) ! !}{(2 n-1) ! !}\right)^{2}\left\{\frac{n^{p}+\sum_{j=1}^{p} a_{j} n^{p-j}}{n^{q}+\sum_{j=1}^{q} b_{j} n^{q-j}}+O\left(\frac{1}{n^{p+q+2}}\right)\right\}, \quad n \rightarrow \infty
$$

where $p \geq 0$ and $q \geq 1$ are two given integers and $q=p+1$, and the coefficients $a_{j}$ and $b_{j}$ are given by (3.7). 
Remark 3.2 Setting $(p, q)=(k, k+1)$ in (3.10) yields $(1.5)$.

Setting

$$
(p, q)=(4,5) \text { and }(p, q)=(5,6)
$$

in (3.10), respectively, we find

$$
\pi=\left(\frac{(2 n) ! !}{(2 n-1) ! !}\right)^{2}\left\{\frac{n^{4}+n^{3}+\frac{107}{64} n^{2}+\frac{91}{128} n+\frac{789}{4,096}}{n^{5}+\frac{5}{4} n^{4}+\frac{125}{64} n^{3}+\frac{295}{256} n^{2}+\frac{1,689}{4,096} n+\frac{945}{16,384}}+O\left(\frac{1}{n^{11}}\right)\right\}
$$

and

$$
\begin{aligned}
\pi= & \left(\frac{(2 n) ! !}{(2 n-1) ! !}\right)^{2} \\
& \times\left\{\frac{n^{5}+\frac{5}{4} n^{4}+\frac{51}{16} n^{3}+\frac{133}{64} n^{2}+\frac{5,243}{4,096} n+\frac{3,867}{16,384}}{n^{6}+\frac{3}{2} n^{5}+\frac{113}{32} n^{4}+\frac{93}{32} n^{3}+\frac{7,729}{4,096} n^{2}+\frac{4,881}{8,192} n+\frac{10,395}{131,072}}+O\left(\frac{1}{n^{13}}\right)\right\}
\end{aligned}
$$

as $n \rightarrow \infty$

Formulas (3.11) and (3.12) motivate us to establish the following theorem.

Theorem 3.2 The following inequality holds:

$$
\begin{gathered}
\frac{x^{5}+\frac{5}{4} x^{4}+\frac{51}{16} x^{3}+\frac{133}{64} x^{2}+\frac{5,243}{4,096} x+\frac{3,867}{16,384}}{x^{6}+\frac{3}{2} x^{5}+\frac{113}{32} x^{4}+\frac{93}{32} x^{3}+\frac{7,729}{4,096} x^{2}+\frac{4,881}{8,192} x+\frac{10,395}{131,072}} \\
<\left(\frac{\Gamma\left(x+\frac{1}{2}\right)}{\Gamma(x+1)}\right)^{2} \\
<\frac{x^{4}+x^{3}+\frac{107}{64} x^{2}+\frac{91}{128} x+\frac{789}{4,096}}{x^{5}+\frac{5}{4} x^{4}+\frac{125}{64} x^{3}+\frac{295}{256} x^{2}+\frac{1,689}{4,096} x+\frac{945}{16,384}} .
\end{gathered}
$$

The left-hand side inequality holds for $x \geq 4$, while the right-hand side inequality is valid for $x \geq 3$.

Proof It suffices to show that

$$
F(x)>0 \quad \text { for } x \geq 4 \text { and } \quad G(x)<0 \quad \text { for } x \geq 3 \text {, }
$$

where

$$
F(x)=2 \ln \left(\frac{\Gamma\left(x+\frac{1}{2}\right)}{\Gamma(x+1)}\right)-\ln \frac{x^{5}+\frac{5}{4} x^{4}+\frac{51}{16} x^{3}+\frac{133}{64} x^{2}+\frac{5,243}{4,096} x+\frac{3,867}{16,384}}{x^{6}+\frac{3}{2} x^{5}+\frac{113}{32} x^{4}+\frac{93}{32} x^{3}+\frac{7,729}{4,096} x^{2}+\frac{4,881}{8,192} x+\frac{10,395}{131,072}}
$$

and

$$
G(x)=2 \ln \left(\frac{\Gamma\left(x+\frac{1}{2}\right)}{\Gamma(x+1)}\right)-\ln \frac{x^{4}+x^{3}+\frac{107}{64} x^{2}+\frac{91}{128} x+\frac{789}{4,096}}{x^{5}+\frac{5}{4} x^{4}+\frac{125}{64} x^{3}+\frac{295}{256} x^{2}+\frac{1,689}{4,096} x+\frac{945}{16,384}} .
$$


Using the following asymptotic expansion (see [12]):

$$
\begin{aligned}
{\left[\frac{\Gamma\left(x+\frac{1}{2}\right)}{\Gamma(x+1)}\right]^{2} \sim } & \frac{1}{x} \exp \left(-\frac{1}{4 x}+\frac{1}{96 x^{3}}-\frac{1}{320 x^{5}}+\frac{17}{7,168 x^{7}}-\frac{31}{9,216 x^{9}}\right. \\
& \left.+\frac{691}{90,112 x^{11}}-\frac{5,461}{212,992 x^{13}}+\frac{929,569}{7,864,320 x^{15}}-\cdots\right), \quad x \rightarrow \infty,
\end{aligned}
$$

we obtain that

$$
\lim _{x \rightarrow \infty} F(x)=0 \quad \text { and } \quad \lim _{x \rightarrow \infty} G(x)=0
$$

Differentiating $F(x)$ and applying the first inequality in (2.6), we find

$$
\begin{aligned}
F^{\prime}(x) & =-2\left[\psi(x+1)-\psi\left(x+\frac{1}{2}\right)\right]+\frac{P_{10}(x)}{P_{11}(x)} \\
& <-2 U(x)+\frac{P_{10}(x)}{P_{11}(x)}=-\frac{P_{16}(x-4)}{524,288 x^{18} P_{11}(x)},
\end{aligned}
$$

where

$$
\begin{aligned}
P_{10}(x)= & 4\left(20,998,323+301,244,208 x+1,329,622,624 x^{2}+3,532,111,872 x^{3}\right. \\
& +6,831,390,720 x^{4}+8,950,906,880 x^{5}+9,510,060,032 x^{6} \\
& \left.+6,476,005,376 x^{7}+4,244,635,648 x^{8}+1,342,177,280 x^{9}+536,870,912 x^{10}\right), \\
P_{11}(x)= & \left(16,384 x^{5}+20,480 x^{4}+52,224 x^{3}+34,048 x^{2}+20,972 x+3,867\right) \\
& \times\left(131,072 x^{6}+196,608 x^{5}+462,848 x^{4}+380,928 x^{3}+247,328 x^{2}\right. \\
& +78,096 x+10,395)
\end{aligned}
$$

and

$$
\begin{aligned}
P_{16}(x)= & 73,399,302,245,132,658,732,474+401,687,666,421,636,714,876,048 x \\
& +882,663,824,965,187,436,960,169 x^{2} \\
& +1,129,813,735,156,766,429,414,420 x^{3} \\
& +975,385,167,000,268,446,720,384 x^{4} \\
& +611,802,531,654,753,268,270,848 x^{5} \\
& +290,696,674,545,996,984,221,376 x^{6} \\
& +107,149,026,028,490,487,475,968 x^{7} \\
& +31,018,031,026,615,120,693,760 x^{8} \\
& +7,080,024,048,117,231,228,928 x^{9} \\
& +1,270,066,473,244,063,756,800 x^{10}+177,136,978,237,041,715,200 x^{11} \\
& +18,824,726,793,935,462,400 x^{12}+1,473,208,721,923,276,800 x^{13}
\end{aligned}
$$




$$
\begin{aligned}
& +80,051,720,723,251,200 x^{14}+2,698,074,228,326,400 x^{15} \\
& +42,489,357,926,400 x^{16}
\end{aligned}
$$

Hence, $F^{\prime}(x)<0$ for $x \geq 4$, and we have

$$
F(x)>\lim _{t \rightarrow \infty} F(t)=0, \quad x \geq 4
$$

Differentiating $G(x)$ and applying the second inequality in (2.6), we find

$$
\begin{aligned}
G^{\prime}(x) & =-2\left[\psi(x+1)-\psi\left(x+\frac{1}{2}\right)\right]+\frac{4 P_{8}(x)}{P_{9}(x)}>-2 V(x)+\frac{4 P_{8}(x)}{P_{9}(x)} \\
& =\frac{P_{14}(x-3)}{524,288 x^{16} P_{9}(x)}
\end{aligned}
$$

where

$$
\begin{aligned}
P_{8}(x)= & 16,777,216 x^{8}+33,554,432 x^{7}+72,351,744 x^{6}+79,167,488 x^{5}+75,583,488 x^{4} \\
& +45,043,712 x^{3}+18,211,328 x^{2}+4,212,480 x+644,661 \\
P_{9}(x)= & \left(4,096 x^{4}+4,096 x^{3}+6,848 x^{2}+2,912 x+789\right) \\
& \times\left(16,384 x^{5}+20,480 x^{4}+32,000 x^{3}+18,880 x^{2}+6,756 x+945\right)
\end{aligned}
$$

and

$$
\begin{aligned}
P_{14}(x)= & 427,884,340,806,856,575+5,508,337,280,234,438,700 x \\
& +16,278,641,070,340,979,232 x^{2} \\
& +25,110,186,749,213,013,376 x^{3}+25,009,399,125,661,680,960 x^{4} \\
& +17,642,792,222,808,253,696 x^{5} \\
& +9,230,356,959,310,493,184 x^{6}+3,661,094,552,739,530,752 x^{7} \\
& +1,108,535,832,992,448,000 x^{8} \\
& +255,024,028,762,675,200 x^{9}+43,854,087,132,979,200 x^{10} \\
& +5,462,018,666,496,000 x^{11} \\
& +465,495,496,704,000 x^{12}+24,287,993,856,000 x^{13} \\
& +585,252,864,000 x^{14} .
\end{aligned}
$$

Hence, $G^{\prime}(x)>0$ for $x \geq 3$, and we have

$$
G(x)<\lim _{t \rightarrow \infty} G(t)=0, \quad x \geq 3 .
$$

The proof is complete. 
Corollary 3.2 For $n \in \mathbb{N}$,

$$
a_{n}<\pi<b_{n}
$$

where

$$
a_{n}=\frac{n^{5}+\frac{5}{4} n^{4}+\frac{51}{16} n^{3}+\frac{133}{64} n^{2}+\frac{5,243}{4,096} n+\frac{3,867}{16,384}}{n^{6}+\frac{3}{2} n^{5}+\frac{113}{32} n^{4}+\frac{93}{32} n^{3}+\frac{7,729}{4,096} n^{2}+\frac{4,881}{8,192} n+\frac{10,395}{131,072}}\left(\frac{(2 n) ! !}{(2 n-1) ! !}\right)^{2}
$$

and

$$
b_{n}=\frac{n^{4}+n^{3}+\frac{107}{64} n^{2}+\frac{91}{128} n+\frac{789}{4,096}}{n^{5}+\frac{5}{4} n^{4}+\frac{125}{64} n^{3}+\frac{295}{256} n^{2}+\frac{1,689}{4,096} n+\frac{945}{16,384}}\left(\frac{(2 n) ! !}{(2 n-1) ! !}\right)^{2} .
$$

Proof Noting that (3.5) holds, we see by (3.13) that the left-hand side of (3.15) holds for $n \geq 4$, while the right-hand side of (3.15) is valid for $n \geq 3$. Elementary calculations show that the left-hand side of (3.15) is also valid for $n=1,2$ and 3 , and the right-hand side of (3.15) is valid for $n=1$ and 2 . The proof is complete.

\section{Comparison}

Recently, Lin [12] improved Mortici's result (1.3) and obtained the following inequalities:

$$
\lambda_{n}<\pi<\mu_{n}
$$

and

$$
\delta_{n}<\pi<\omega_{n}
$$

where

$$
\begin{aligned}
\lambda_{n}= & \left(1+\frac{1}{4 n}-\frac{3}{32 n^{2}}+\frac{3}{128 n^{3}}+\frac{3}{2,048 n^{4}}-\frac{33}{8,192 n^{5}}-\frac{39}{65,536 n^{6}}\right) \\
& \times \frac{2}{2 n+1}\left(\frac{(2 n) ! !}{(2 n-1) ! !}\right)^{2}, \\
\mu_{n}= & \left(1+\frac{1}{4 n}-\frac{3}{32 n^{2}}+\frac{3}{128 n^{3}}+\frac{3}{2,048 n^{4}}\right) \frac{2}{2 n+1}\left(\frac{(2 n) ! !}{(2 n-1) ! !}\right)^{2}, \\
\delta_{n}= & \left(\frac{(2 n) ! !}{(2 n-1) ! !}\right)^{2} \frac{1}{n} \exp \left(-\frac{1}{4 n}+\frac{1}{96 n^{3}}-\frac{1}{320 n^{5}}+\frac{17}{7,168 n^{7}}-\frac{31}{9,216 n^{9}}\right), \\
\omega_{n}= & \left(\frac{(2 n) ! !}{(2 n-1) ! !}\right)^{2} \frac{1}{n} \exp \left(-\frac{1}{4 n}+\frac{1}{96 n^{3}}-\frac{1}{320 n^{5}}+\frac{17}{7,168 n^{7}}\right) .
\end{aligned}
$$

Direct computation yields

$$
\begin{aligned}
a_{n}- & \lambda_{n} \\
= & \frac{3\left(7,634,944 n^{5}+12,928,000 n^{4}+18,895,616 n^{3}+9,755,072 n^{2}+1,930,008 n+135,135\right)}{32,768 n^{6}(2 n+1)\left(131,072 n^{6}+196,608 n^{5}+462,848 n^{4}+380,928 n^{3}+247,328 n^{2}+78,096 n+10,395\right)} \\
& \times\left(\frac{(2 n) ! !}{(2 n-1) ! !}\right)^{2}>0
\end{aligned}
$$


Table 1 Comparison between inequalities (3.15) and (4.2)

\begin{tabular}{rll}
\hline $\boldsymbol{n}$ & $\boldsymbol{a}_{\boldsymbol{n}}-\boldsymbol{\delta}_{\boldsymbol{n}}$ & $\boldsymbol{\omega}_{\boldsymbol{n}}-\boldsymbol{b}_{\boldsymbol{n}}$ \\
\hline 1 & $6.673798 \times 10^{-3}$ & $3.789512 \times 10^{-3}$ \\
10 & $2.264856 \times 10^{-13}$ & $9.947434 \times 10^{-12}$ \\
100 & $2.398663 \times 10^{-24}$ & $1.051407 \times 10^{-20}$ \\
1,000 & $2.408054 \times 10^{-35}$ & $1.056218 \times 10^{-29}$ \\
10,000 & $2.408948 \times 10^{-46}$ & $1.056690 \times 10^{-38}$ \\
\hline
\end{tabular}

and

$$
\begin{aligned}
b_{n} & -\mu_{n} \\
& =-\frac{3\left(45,056 n^{4}+62,976 n^{3}+66,496 n^{2}+21,876 n+945\right)}{1,024 n^{4}(2 n+1)\left(16,384 n^{5}+20,480 n^{4}+32,000 n^{3}+18,880 n^{2}+6,756 n+945\right)}\left(\frac{(2 n) ! !}{(2 n-1) ! !}\right)^{2} \\
& <0 .
\end{aligned}
$$

Hence, (3.15) improves (4.1).

The following numerical computations (see Table 1) would show that $\delta_{n}<a_{n}$ and $b_{n}<\omega_{n}$ for $n \in \mathbb{N}$. That is to say, inequalities (3.15) are sharper than inequalities (4.2).

In fact, we have

$$
\begin{array}{ll}
\lambda_{n}=\pi+O\left(\frac{1}{n^{7}}\right), & \mu_{n}=\pi+O\left(\frac{1}{n^{5}}\right), \\
\delta_{n}=\pi+O\left(\frac{1}{n^{11}}\right), & \omega_{n}=\pi+O\left(\frac{1}{n^{9}}\right), \\
a_{n}=\pi+O\left(\frac{1}{n^{12}}\right), & b_{n}=\pi+O\left(\frac{1}{n^{10}}\right) .
\end{array}
$$

\section{Acknowledgements}

The authors thank the referees for helpful comments.

\section{Competing interests}

The authors declare that they have no competing interests.

\section{Authors' contributions}

All authors read and approved the final manuscript.

\section{Author details}

'School of Mathematics and Informatics, Henan Polytechnic University, Jiaozuo City, Henan Province 454000, China. ${ }^{2}$ College of Chemistry and Chemical Engineering, Henan Polytechnic University, Jiaozuo City, Henan Province 454000 China.

\section{Publisher's Note}

Springer Nature remains neutral with regard to jurisdictional claims in published maps and institutional affiliations.

Received: 17 March 2017 Accepted: 15 May 2017 Published online: 08 June 2017

\section{References}

1. Gurland, J: On Wallis' formula. Am. Math. Mon. 63, 643-645 (1956)

2. Brutman, L: A sharp estimate of the Landau constants. J. Approx. Theory 34, 217-220 (1982)

3. Falaleev, LP: Inequalities for the Landau constants. Sib. Math. J. 32, 896-897 (1991)

4. Mortici, C: Refinements of Gurland's formula for pi. Comput. Math. Appl. 62, 2616-2620 (2011)

5. Chen, CP, Paris, RB: Inequalities, asymptotic expansions and completely monotonic functions related to the gamma function. Appl. Math. Comput. 250, 514-529 (2015)

6. Bercu, G: Padé approximant related to remarkable inequalities involving trigonometric functions. J. Inequal. Appl. 2016, 99 (2016)

7. Bercu, G: The natural approach of trigonometric inequalities-Padé approximant. J. Math. Inequal. 11, 181-191 (2017) 
8. Bercu, G, Wu, S: Refinements of certain hyperbolic inequalities via the Padé approximation method. J. Nonlinear Sci. Appl. 9, 5011-5020 (2016)

9. Brezinski, C, Redivo-Zaglia, M: New representations of Padé, Padé-type, and partial Padé approximants. J. Comput. Appl. Math. 284, 69-77 (2015)

10. Li, X, Chen, CP: Padé approximant related to asymptotics for the gamma function. J. Inequal. Appl. 2017, 53 (2017)

11. Liu, J, Chen, CP: Padé approximant related to inequalities for Gauss lemniscate functions. J. Inequal. Appl. 2016, 320 (2016)

12. Lin, L: Further refinements of Gurland's formula for $\pi$. J. Inequal. Appl. 2013, 48 (2013)

Submit your manuscript to a SpringerOpen ${ }^{\circ}$ journal and benefit from:

- Convenient online submission

- Rigorous peer review

- Immediate publication on acceptance

Open access: articles freely available online

- High visibility within the field

- Retaining the copyright to your article 